\title{
Autologous Dendritic Cell-based Immunotherapeutic AV0113
}

National Cancer Institute

\section{Source}

National Cancer Institute. Autologous Dendritic Cell-based Immunotherapeutic AV0113.

NCl Thesaurus. Code C123928.

A therapeutic interleukin-12 (IL-12)-expressing dendritic cell (DC)-based vaccine composed of autologous monocyte-derived DCs loaded with autologous tumor cell lysate and exposed to the microbial cell wall component lipopolysaccharide (LPS), with potential immunomodulating and antineoplastic activities. The monocyte-derived immature DCs are loaded with autologous tumor cell lysates and are subsequently exposed to LPS and interferon-gamma (IFN-gamma). Upon administration of autologous DC-based immunotherapeutic AV0113, the mature DCs migrate into the lymph nodes, express the immune stimulatory cytokine interleukin-12 (IL-12) and activate the immune system by promoting the activation of natural killer (NK) cells and induce a cytotoxic T-lymphocyte (CT L)-mediated immune response against tumor cells, which may result in immune-mediated tumor cell death and inhibition of tumor cell proliferation. Exposure to LPS and IFN-gamma allows the maturation of DCs and optimizes the presentation of tumor-associated antigens (TAAs) by DCs to T-lymphocytes. 